\title{
Impact of tumor size on outcomes after anatomic lung resection for stage 1A non-small cell lung cancer based on the current staging system
}

\author{
Shamus R. Carr, MD, ${ }^{\mathrm{a}}$ Matthew J. Schuchert, MD, ${ }^{\mathrm{a}}$ Arjun Pennathur, MD, ${ }^{\mathrm{a}}$ David O. Wilson, MD, \\ Jill M. Siegfried, $\mathrm{PhD},{ }^{\mathrm{c}}$ James D. Luketich, $\mathrm{MD},{ }^{\mathrm{a}}$ and Rodney J. Landreneau, $\mathrm{MD}^{\mathrm{a}}$
}

\begin{abstract}
Objective: Anatomic segmentectomy may achieve results comparable to lobectomy for early-stage non-small cell lung cancer. The 7th edition of the AJCC Cancer Staging Handbook stratified the previous T1 tumor designation into T1a and T1b subsets, which still define stage 1A node-negative non-small cell lung cancer. We are left to hypothesize whether this classification may aid in directing the extent of surgical resection. We retrospectively reviewed our anatomic segmentectomy and lobectomy management of stage $1 \mathrm{~A}$ non-small cell lung cancer to determine differences in survival and local recurrence rates based on the new stratification.
\end{abstract}

\begin{abstract}
Methods: We performed a retrospective review of 429 patients undergoing resection of pathologically confirmed stage 1A non-small cell lung cancer via lobectomy or anatomic segmentectomy. Primary outcome variables included mortality, recurrence, and survival. Recurrence-free and cancer-specific survivals were estimated using the Kaplan-Meier method.
\end{abstract}

Results: Patients undergoing segmentectomy were older than patients undergoing lobectomy (mean age 69.2 vs 66.8 years, $P<.006$ ). The mean preoperative forced expiratory volume in 1 second was significantly lower in the segmentectomy group than in the lobectomy group $(71.8 \%$ vs $81.1 \%, P=.02)$. Mortality was similar after segmentectomy $(1.1 \%)$ and lobectomy $(1.2 \%)$. There was no difference in mortality, recurrence rates $(14.0 \% \mathrm{vs}$ $14.7 \%, P=1.00$ ), or 5 -year cancer-specific survival (T1a: $90 \%$ vs $91 \%, P=.984$; T1b: $82 \%$ vs $78 \%$, $P=.892$ ) when comparing segmentectomy and lobectomy for pathologic stage 1 A non-small cell lung cancer, when stratified by $\mathrm{T}$ stage.

Conclusions: Anatomic segmentectomy may achieve equivalent recurrence and survival compared with lobectomy for patients with stage 1A non-small cell lung cancer. Prospective studies will be necessary to delineate the potential merits of anatomic segmentectomy in this setting. (J Thorac Cardiovasc Surg 2012;143:390-7)

The new international lung cancer staging system is the culmination of a tremendous comprehensive effort of thoracic surgeons, oncologists, pulmonary specialists, radiologists, pathologists, and statisticians across the globe. The statistically validated multi-year retrospective analyses of the results of therapy of more than 100,000 patients with lung cancer by the International Association for the Study of

From the Division of Thoracic and Foregut Surgery, ${ }^{\mathrm{a}}$ Department of Cardiothoracic Surgery, Division of Pulmonary, Allergy, and Critical Care Medicine, ${ }^{b}$ and Department of Pharmacology and Chemical Biology, ${ }^{\mathrm{c}}$ University of Pittsburgh, Pittsburgh, Pa.

Funding: Heart, Lung, and Esophageal Surgery Institute, UPMC Health System, Pittsburgh, Pennsylvania.

Disclosures: Authors have nothing to disclose with regard to commercial support.

Read at the 37th Annual Meeting of The Western Thoracic Surgical Association, Colorado Springs, Colorado, June 22-25, 2011.

Received for publication June 29, 2010; revisions received Aug 31, 2011; accepted for publication Oct 20, 2011; available ahead of print Dec 12, 2011.

Address for reprints: Matthew J. Schuchert, MD, Department of Cardiothoracic Surgery, Shadyside Medical Building, Suite 715, 5200 Centre Avenue, Pittsburgh, PA 15232 (E-mail: schuchertmj@upmc.edu).

0022-5223/ $\$ 36.00$

Copyright (C) 2012 by The American Association for Thoracic Surgery doi: $10.1016 /$ j.jtcvs.2011.10.023
Lung Cancer have led to a comprehensive, detailed staging system. $^{1,2}$ The cooperation of the International Union Against Cancer and American Joint Committee on Cancer (AJCC) resulted in the publication of a uniform staging manual accepted worldwide: the 7th edition of the AJCC Cancer Staging Handbook. ${ }^{3}$

Although lobectomy is considered by many surgeons to be the first line of therapy for stage 1 non-small cell lung cancer (NSCLC), ${ }^{4}$ a growing body of evidence suggests an acceptable and possible preferable role of anatomic segmentectomy for the definitive management of small peripheral stage I NSCLC. ${ }^{5-7}$ Use of sublobar resection, preferably anatomic segmentectomy, in lieu of lobectomy for the management of otherwise resectable stage 1 NSCLC identified in patients of advanced age or with impaired cardiopulmonary reserve is also a reasonable resection consideration. ${ }^{5-11}$

The 7th edition of the AJCC Cancer Staging Handbook for NSCLC has subdivided stage 1A tumors $(\leq 3 \mathrm{~cm})$ into 2 subsets: T1a $(\leq 2 \mathrm{~cm})$ and T1b $(>2 \mathrm{~cm}$ and $\leq 3 \mathrm{~cm}) .{ }^{3} \mathrm{By}$ using this new stage $1 \mathrm{~A}$ subclassification system, we analyzed the clinical outcomes of patients with pathologically confirmed stage 1A NSCLC who underwent R0 resection 


\section{Abbreviations and Acronyms}

AJCC $=$ American Joint Committee on Cancer

$\mathrm{FEV}_{1}=$ forced expiratory volume in 1 second

NSCLC $=$ non-small cell lung cancer

VATS $=$ video-assisted thoracoscopic surgery

by anatomic segmentectomy or lobectomy from 2002 to 2009. Our goals were to determine whether differences could be determined in recurrence and survival patterns between patients with stage 1A NSCLC definitively managed by anatomic segmentectomy or lobectomy based on the new stratification in the T stage: T1a and T1b.

\section{PATIENTS AND METHODS}

\section{Patient Selection and Surgical Approach}

This study was reviewed and approved by the institutional review board of the University of Pittsburgh (No. PRO009120047), and individual patient consent was waived given the retrospective nature of the analysis. A total of 429 consecutive patients with anatomic pulmonary resection for pathologically confirmed stage 1A NSCLC from 2002 to 2009 were identified by the Thoracic Tumor Registry and from the billing records of the Heart, Lung, and Esophageal Surgery Institute of the University of Pittsburgh Medical Center. Only patients with pathologic stage 1A disease were included in this study (Table 1). Lobectomy was performed in 251 patients, by thoracotomy in 152 and video-assisted thoracoscopic surgery (VATS) in 99. Anatomic segmentectomy was performed in 178 patients. Of these patients, 73 underwent open segmentectomy and 105 patients underwent anatomic segmentectomy via a VATS approach. All patients underwent careful preoperative staging with computed tomography scan (with or without positron emission tomography) within 6 weeks of surgery and pulmonary function testing. Positron emission tomography scanning was not routinely performed in this cohort of patients with early-stage disease but was selectively used according to tumor size and location, evidence for mediastinal lymph node enlargement on computed tomography imaging, and physician judgment. Mediastinoscopy was performed in patients with enlarged mediastinal lymph nodes $(>1 \mathrm{~cm}$ in diameter) or in patients with positive FDG uptake on positron emission tomography scanning. A total of 21 of 178 patients $(11.8 \%)$ in the segmentectomy group and 50 of 251 patients $(19.9 \%)$ in the lobectomy group underwent mediastinoscopy. All patients were staged on final pathologic review as stage 1A according to the TNM classification of the American Joint Committee for Cancer Staging and the Revised International System for staging lung cancer. ${ }^{3}$

\section{Operative Technique and Hospital Course}

Thoracotomy was performed in 225 patients $(52.4 \%$ ), with a VATS approach used in 204 patients $(47.6 \%)$. All of the principal anatomic segments were resected (Table 2). The largest determinants in selecting a patient for segmentectomy were limited functional status, comorbidities, and surgeon judgment regarding resectability using a segmental approach. However, with time, even patients with good pulmonary function were considered for segmentectomy as long as anatomic considerations were still favorable. Open segmental resection was performed by muscle-sparing axillary thoracotomy or posterolateral thoracotomy. The VATS approach was performed as described previously. ${ }^{7}$ Anatomic segmentectomy was performed with individual isolation and division of the corresponding artery and bronchus. In addition, an extended parenchymal margin was obtained during stapling, which carried the line of division into the
TABLE 1. Patient demographics and operative data by $T$ descriptor

\begin{tabular}{lccc}
\hline & T1a $(\mathbf{n = 2 8 4})$ & T1b $(\mathbf{n}=\mathbf{1 4 5})$ & $\boldsymbol{P}$ value \\
\hline Age, y & & & \\
Mean & 67.0 & 69.4 & .009 \\
$\quad$ Range & $28-88$ & $43-88$ & \\
Gender & 123 male, 161 female & 75 male, 70 female & .10 \\
Operation & & & \\
$\quad$ Segmentectomy & $121(42.6 \%)$ & $57(39.3 \%)$ & .54 \\
$\quad$ Lobectomy & $163(57.4 \%)$ & $88(60.7 \%)$ & \\
Approach & & & \\
$\quad$ VATS & $142(50.0 \%)$ & $62(42.8 \%)$ & .18 \\
$\quad$ Open & $142(50.0 \%)$ & $83(57.2 \%)$ & \\
\hline
\end{tabular}

VATS, Video-assisted thoracoscopic surgery.

adjacent segmental parenchyma (extended segmentectomy). We prefer the stapled, extended approach to segmentectomy, which serves to incorporate the corresponding intersegmental veins (rather than a selective dissection and identification of the intersegmental veins) to minimize the risk of bleeding and prolonged postoperative air leaks. ${ }^{4}$ Systematic mediastinal lymph node sampling is performed during the course of resection. Patients were typically monitored in the intensive care unit overnight and transferred to the floor the following day. Chest tube management and discharge planning were individualized on the basis of patient clinical characteristics and surgeon judgment. Chest tubes were usually removed when air leaks ceased and drainage decreased to less than $250 \mathrm{~mL} /$ day. Patients were discharged after chest tube removal when clinically stable. In cases of prolonged air leaks, patients were discharged after placing a Heimlich valve and ensuring stability of lung expansion. Discharge criteria were not influenced by clinical pathways or type of surgery performed.

\section{Follow-up}

Perioperative and follow-up data were collected from the hospital chart, anesthesia, and operating room records, as well as the electronic medical record for each patient. All patients were followed postoperatively at 2 weeks, at 4- to 6-month intervals for the first 2 years, and yearly thereafter with computed tomography scans. Locoregional recurrence was defined as evidence of tumor within the same lobe, the hilum, or the mediastinal lymph nodes. Distant recurrences were defined as evidence of tumor in another lobe or elsewhere outside the hemithorax. In addition to the data derived from the electronic medical record and the University of Pittsburgh

TABLE 2. Resections performed

\begin{tabular}{lc}
\hline Location & No. of resections performed \\
\hline Right upper lobe & 99 \\
Anterior & 8 \\
Posterior & 18 \\
Apical & 11 \\
Apico-posterior & 8 \\
Right middle lobe & 12 \\
Medial/lateral & 19 \\
Right lower lobe & 39 \\
Superior & 21 \\
Basilar & 12 \\
Left upper lobe & 68 \\
Upper division & 43 \\
Lingula & 14 \\
Left lower lobe & 33 \\
Superior & 13 \\
Basilar & 11 \\
\hline
\end{tabular}


Lung Cancer Registry, mortality data were obtained from the Social Security Death Index. The primary end points for this analysis were perioperative outcomes, morbidity and mortality rates, and disease-free and cancer-specific survival. Perioperative mortality was defined as any patient who died within the first 30 days after surgery. Mean follow-up was 45.3 months for the entire group of patients with stage 1A NSCLC (segmentectomy, 39.6 months; lobectomy, 50.9 months).

\section{Statistical Analysis}

Statistical analyses were performed using the Statistical Package for the Social Sciences 17.0 (SPSS Inc, Chicago, Ill). The lobectomy and segmentectomy groups, based on T stage, were compared with the nonparametric Wilcoxon test for continuous variables and Fisher exact test for categoric variables. Disease-free and overall survival analyses were carried out by means of the Kaplan-Meier approach (log-rank test). All comparisons were 2-tailed. Recurrence-free survival was defined as the time from surgery to the first diagnosis of local, regional, or distant disease recurrence or to the last-follow-up. Cancer-specific survival was defined as overall time of survival to death or last follow-up based on the presence or absence of cancer at the time of death.

\section{RESULTS}

\section{Demographic and Group Characteristics}

The average age of all patients was 67.8 years. The mean age was 67.0 years for patients with T1a tumors and 69.4 years for patients with $\mathrm{T} 1 \mathrm{~b}$ tumors $(P=.009)$ (Table 1). The average tumor size was $1.8 \pm 0.7 \mathrm{~cm}$ for all patients. The mean tumor size was $1.4 \pm 0.4 \mathrm{~cm}$ for T1a tumors and 2.6 $\pm 0.3 \mathrm{~cm}$ for T1b tumors. The mean surgical margin was $1.9 \mathrm{~cm}$ for segments and 2.0 for lobes. All typically resected lobes and segments were represented in the groups (Table 2).

\section{Segmentectomy Versus Lobectomy for T1a and T1b Tumors}

Table 3 summarizes the patient populations who underwent lobectomy or segmentectomy. A total of 429 patients with stage 1A NSCLC underwent anatomic resection. There were 251 lobectomies, of which 99 were VATS and 152 were open lobectomies. In addition, 178 patients underwent anatomic segmentectomy, by a thoracoscopic approach in 105 and an open approach in 73. An open approach was more commonly used with lobectomy than with segmentectomy $(60.6 \%$ vs $41.0 \%, P=.0001)$.

Patients undergoing segmentectomy were more likely to have coronary artery disease ( $18.5 \%$ vs $12.8 \%, P=.036)$ or chronic obstructive pulmonary disease $(26.4 \%$ vs $14.4 \%, P=.0001$ ) compared with lobectomy. Patients undergoing segmentectomy also had significantly worse forced expiratory volume in 1 second $\left(\mathrm{FEV}_{1}\right)$ compared with those undergoing lobectomy $(81.1 \pm 17.6$ vs $71.8 \pm$ $25.6, P=.02)$. Other comorbid conditions were not significantly different between patients undergoing lobectomy and segmentectomy (Table 3).

TABLE 3. Segmentectomy versus lobectomy for stage $1 \mathrm{~A}$ tumors

\begin{tabular}{|c|c|c|c|}
\hline & Segmentectomy $(n=178)$ & Lobectomy $(\mathbf{n}=\mathbf{2 5 1})$ & $P$ value \\
\hline \multicolumn{4}{|l|}{ Comorbidities (\%) } \\
\hline Coronary artery disease & 18.5 & 12.8 & .036 \\
\hline Hypertension & 28.1 & 27.2 & .4 \\
\hline Diabetes & 11.2 & 9.7 & .4 \\
\hline COPD & 26.4 & 14.4 & .0001 \\
\hline \multicolumn{4}{|c|}{ Pulmonary function tests (preoperative) } \\
\hline $\mathrm{FEV}_{1} \pm \mathrm{SD}(\%)$ & $71.8 \pm 25.6$ & $81.1 \pm 17.6$ & .02 \\
\hline Range & $(15 \%-114 \%)$ & $(58 \%-119 \%)$ & \\
\hline $\mathrm{DLCO} \pm \mathrm{SD}(\%)$ & $65.4 \pm 22.4$ & $70.7 \pm 17.7$ & .4 \\
\hline Range & $(15 \%-135 \%)$ & $(35 \%-170 \%)$ & \\
\hline \multicolumn{4}{|l|}{ Approach $(\%)$} \\
\hline VATS & 59.0 & 39.4 & .0001 \\
\hline Open & 41.0 & 60.6 & \\
\hline \multicolumn{4}{|l|}{ Histology (\%) } \\
\hline Adenocarcinoma & 51.7 & 55.0 & .56 \\
\hline Squamous & 36.5 & 30.3 & .18 \\
\hline Bronchoalveolar & 5.1 & 8.0 & .33 \\
\hline Other & 6.7 & 6.7 & 1.00 \\
\hline \multicolumn{4}{|l|}{ Differentiation $(\%)$} \\
\hline Well & 12.9 & 13.1 & 1.00 \\
\hline Moderately & 60.1 & 64.1 & .42 \\
\hline Poorly & 27.0 & 22.7 & .36 \\
\hline Angiolymphatic invasion $(\%)$ & 22.5 & 21.5 & .81 \\
\hline LN harvested (median) & 5 & 12 & $<.001$ \\
\hline LN stations sampled (median) & 4 & 4 & 1.00 \\
\hline Mortality (30 d) & $2(1.1 \%)$ & $3(1.2 \%)$ & 1.00 \\
\hline
\end{tabular}

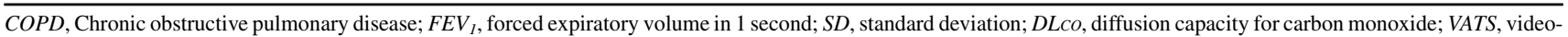
assisted thoracoscopic surgery; $L N$, lymph node. 


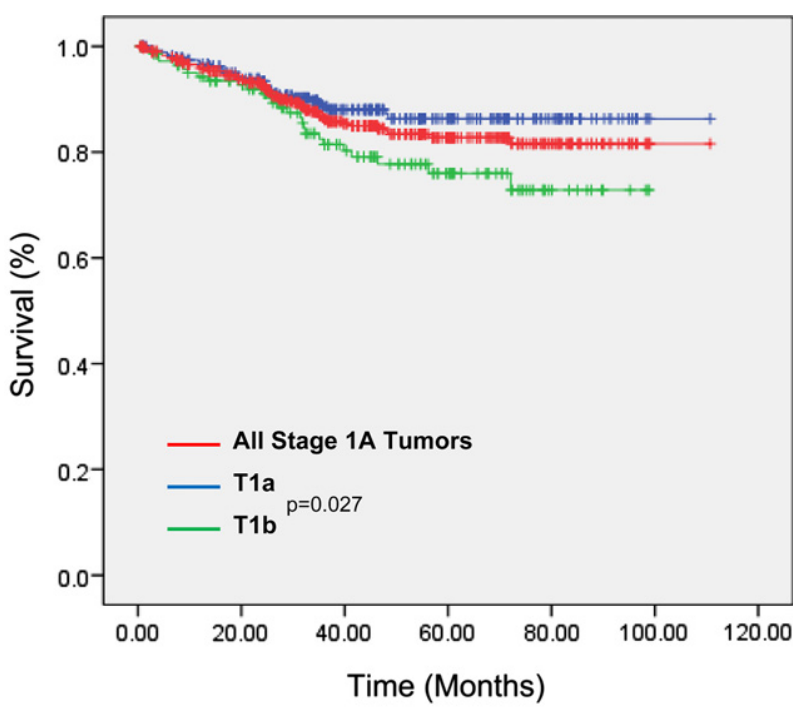

FIGURE 1. Recurrence-free survival of all patients undergoing resection of pathologic stage 1A NSCLC (red). A significant difference in survival is noted when comparing T1a (blue) and T1b (green) tumors $(P=.027)$.

As expected, lobectomy was associated with significantly more lymph nodes examined compared with segmentectomy (median, 12 vs $5 ; P<.0001$ ). However, there was no difference in the number of lymph node stations sampled (median of 4 for both segments and lobes). For T1a tumors, the margin distance:tumor size ratio was $1.52 \pm 1.18$ for segmentectomy and $1.63 \pm 1.28$ for lobectomy $(P=.58)$. For T1b tumors, the margin distance:tumor size ratio was $0.71 \pm 049$ after segmentectomy and $0.80 \pm 0.70$ $(P=.54)$ after lobectomy. These data suggest that the

\section{Segmentectomy}

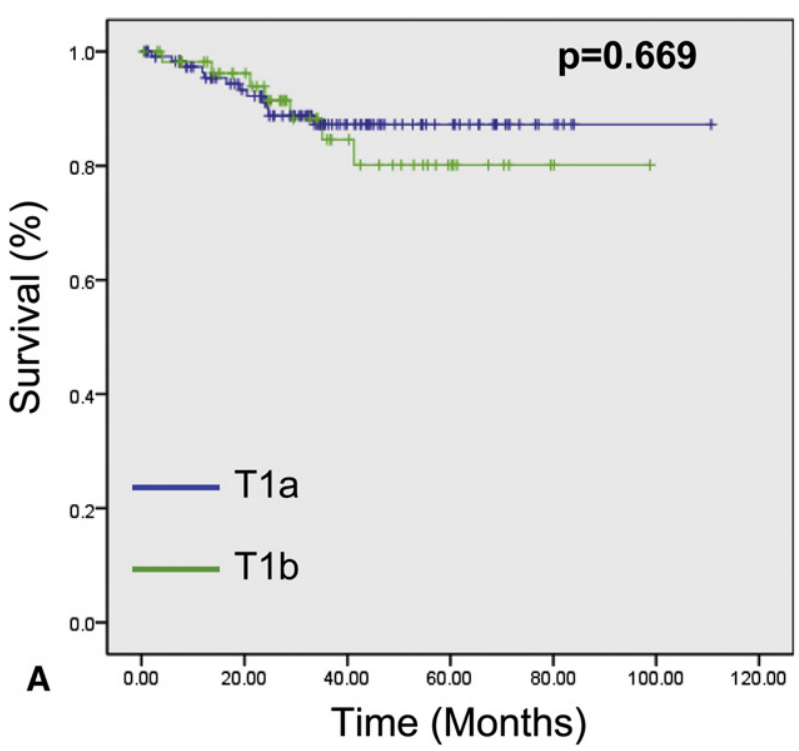

margin:tumor ratio was similar whether performing an anatomic segmentectomy or lobectomy for T1a or T1b tumors. Overall mortality was $1.1 \%$ for segments and $1.2 \%$ for lobes.

\section{Recurrence and Survival}

The mean follow-up in this study was 45.3 months. For all patients undergoing resection, the estimated recurrence-free survival was $82 \%$ at 60 months. When evaluated by $\mathrm{T}$ stage, patients with T1a tumors had an $86 \%$ recurrence-free survival, whereas patients with $\mathrm{T} 1 \mathrm{~b}$ tumors had an estimated 78\% 5-year survival $(P=.027)$ (Figure 1). Recurrence-free survival for those with $\mathrm{T} 1 \mathrm{a}$ and $\mathrm{T} 1 \mathrm{~b}$ tumors stratified by operation type is shown in Figure 2. There was no difference in recurrence-free survival after segmentectomy or lobectomy for those with T1a $(P=.757)$ or T1b $(P=.423)$ tumors (Figure 3$)$. When analyzing patterns of recurrence, there was no significant difference in locoregional or distant recurrence for $\mathrm{T} 1 \mathrm{a}$ or $\mathrm{T} 1 \mathrm{~b}$ tumors (Table 4). Mean time to recurrence was 23 months among all patients. There was an approximately $2: 1$ ratio of distant to locoregional recurrence noted. Brachytherapy mesh was used in 54 of 178 patients (30.3\%) undergoing segmentectomy. The use of brachytherapy mesh was not associated with any discernible difference in locoregional $(5.6 \%$ vs $5.6 \%)$, distant $(9.3$ vs $8.9 \%)$, or overall $(14.8 \%$ vs $14.5 \%)$ recurrence rates compared with patients undergoing segmentectomy without mesh $(P=1.00)$.

When considering deaths related to cancer, there was no significant difference in cancer-specific survival when comparing segmentectomy with lobectomy for T1a or

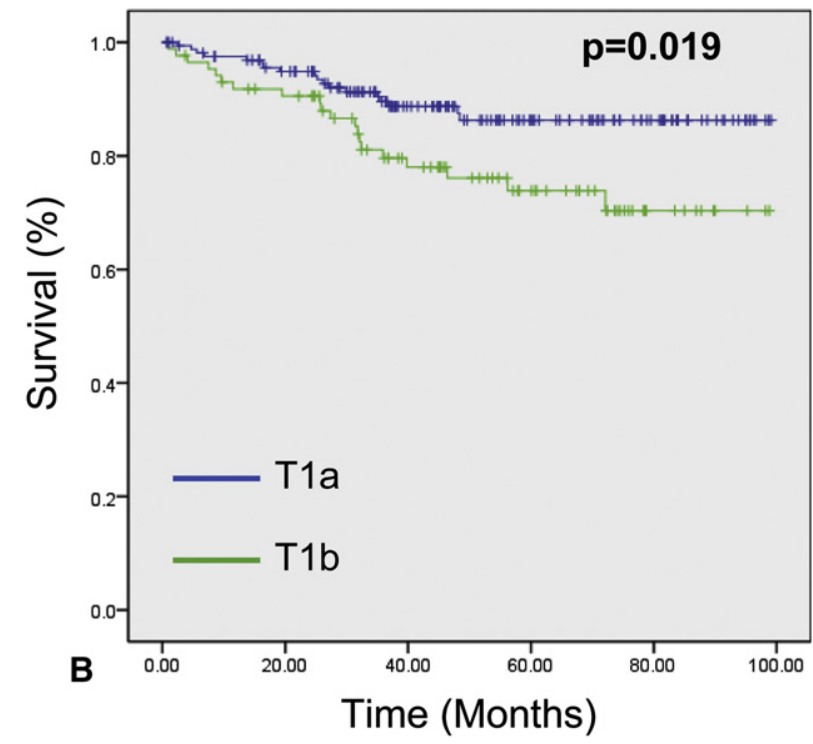

FIGURE 2. Recurrence-free survival for T1a and T1b tumors stratified by segmentectomy (A) and lobectomy (B). 
T1a $(\leq 2 \mathrm{~cm})$

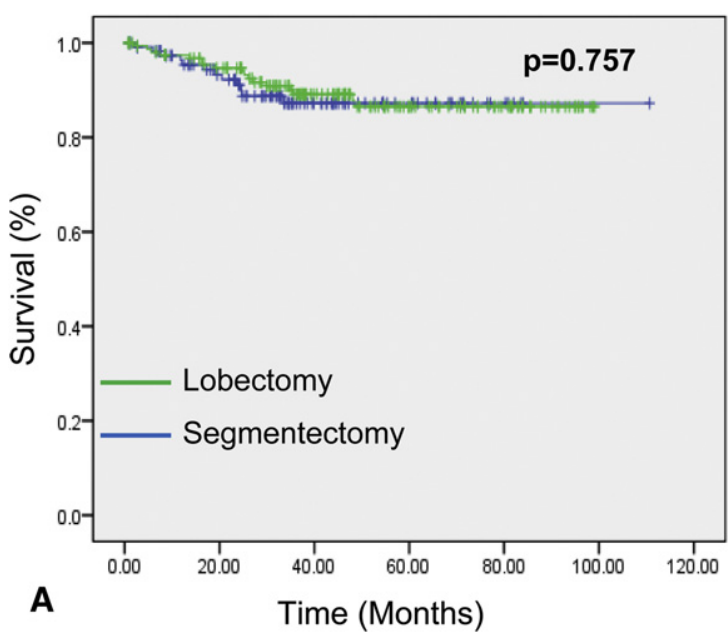

$\mathrm{T} 1 \mathrm{~b}(>2$ and $\leq 3 \mathrm{~cm})$

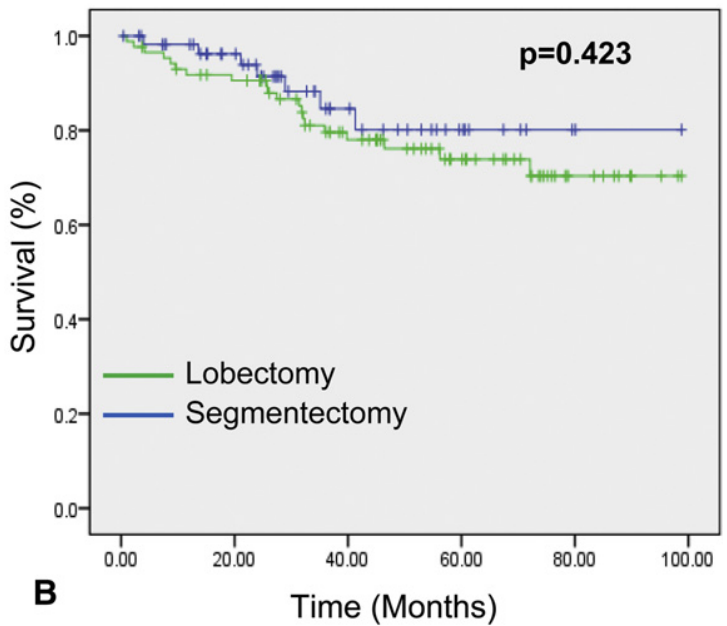

FIGURE 3. Recurrence-free survival curves comparing outcomes after segmentectomy or lobectomy for T1a (A) and T1b (B) tumors.

T1b tumors (Figure 4). Of note, segmentectomies were associated with a significantly increased rate of non-cancerrelated deaths compared with lobectomy, $79 \%$ versus $56 \%$, respectively $(P=.0065)$, perhaps because patients undergoing segmentectomy had greater preoperative comorbidity (coronary artery disease, chronic obstructive pulmonary disease, decreased $\mathrm{FEV}_{1}$ ) when compared with those undergoing lobectomy (Figure 4).

\section{DISCUSSION}

Many patients referred to the thoracic surgeon are sent for definitive management of the small peripheral pulmonary lesion highly suspicious for carcinoma or with a previous image-guided diagnosis of lung cancer. On review of

TABLE 4. Recurrence and survival patterns

\begin{tabular}{lccc}
\hline & $\begin{array}{c}\text { Segmentectomy } \\
(\mathbf{n}=\mathbf{1 2 1})\end{array}$ & $\begin{array}{c}\text { Lobectomy } \\
(\mathbf{n}=\mathbf{1 6 3})\end{array}$ & $\boldsymbol{P}$ value \\
\hline T1a & & & \\
$\quad$ Disease-free & $104(86.0 \%)$ & $139(85.3 \%)$ & 1.00 \\
Overall recurrences & $17(14.0 \%)$ & $24(14.7)$ & 1.00 \\
Locoregional & $7(5.8 \%)$ & $7(4.3 \%)$ & .59 \\
Distant & $10(8.3 \%)$ & $17(10.4 \%)$ & .68 \\
Mean time to recurrence & 19.7 & 23.8 & .39 \\
\hline & Segmentectomy & Lobectomy & \\
& $(\mathbf{n}=\mathbf{5 7})$ & $(\mathbf{n}=\mathbf{8 8})$ & $\boldsymbol{P}$ value \\
\hline T1b & & & \\
Disease-free & $48(84.2 \%)$ & $62(64.7 \%)$ & .074 \\
Overall & $9(15.8 \%)$ & $26(29.5 \%)$ & \\
Locoregional & $3(5.3 \%)$ & $8(9.0 \%)$ & .53 \\
Distant & $6(10.5 \%)$ & $18(20.5 \%)$ & .17 \\
Mean time to recurrence & 28.9 & 23.4 & .58 \\
\hline \multicolumn{3}{c}{}
\end{tabular}

the pulmonary anatomy and pathology, many thoracic surgeons question the need for lobar resection of small lesions, which may be easily resected with a "margin free" sublobar approach. Certainly, image-guided interventionalists (ie, interventional radiologists, radiation oncologists) and many prospective patients are intrigued with the possibility of less drastic/morbid clinically competitive ablative measures that may be associated with preservation of normal pulmonary parenchyma and potential clinical equivalency to lobectomy.

The possible role of anatomic segmentectomy as an alternative to lobectomy in this clinical setting is intriguing and certainly clinically competitive to image-guided ablative approaches, particularly when segmentectomy can be done using minimally invasive surgical approaches. ${ }^{7,12}$ In addition, we should consider the primary arguments in favor of a surgical resection of the small stage 1A lung cancer over image-guided ablative approaches. These include complete resection of the cancer with clear surgical margins determined by pathologic review; the ability to supplement the local control of the disease with intraoperative brachytherapy when surgical margins are $\operatorname{close}^{13}$; comprehensive lymph node staging of the malignancy to avoid understaging of the disease and loss of the opportunity for adjuvant systemic therapy for these understaged patients; and pharmacogenomic testing of the tumor mass to provide enhanced individualized systemic adjuvant therapy of the patient's disease.

The use of segmentectomy for the management of earlystage NSCLC is not a new concept. Indeed, segmentectomy for benign pulmonary conditions dates back several years. ${ }^{14}$ The use of this approach for early-stage NSCLC, first reported in 1973 by Jensik and associates,${ }^{15}$ was supported by others over the ensuing 2 decades. ${ }^{16,17}$ Unfortunately, 

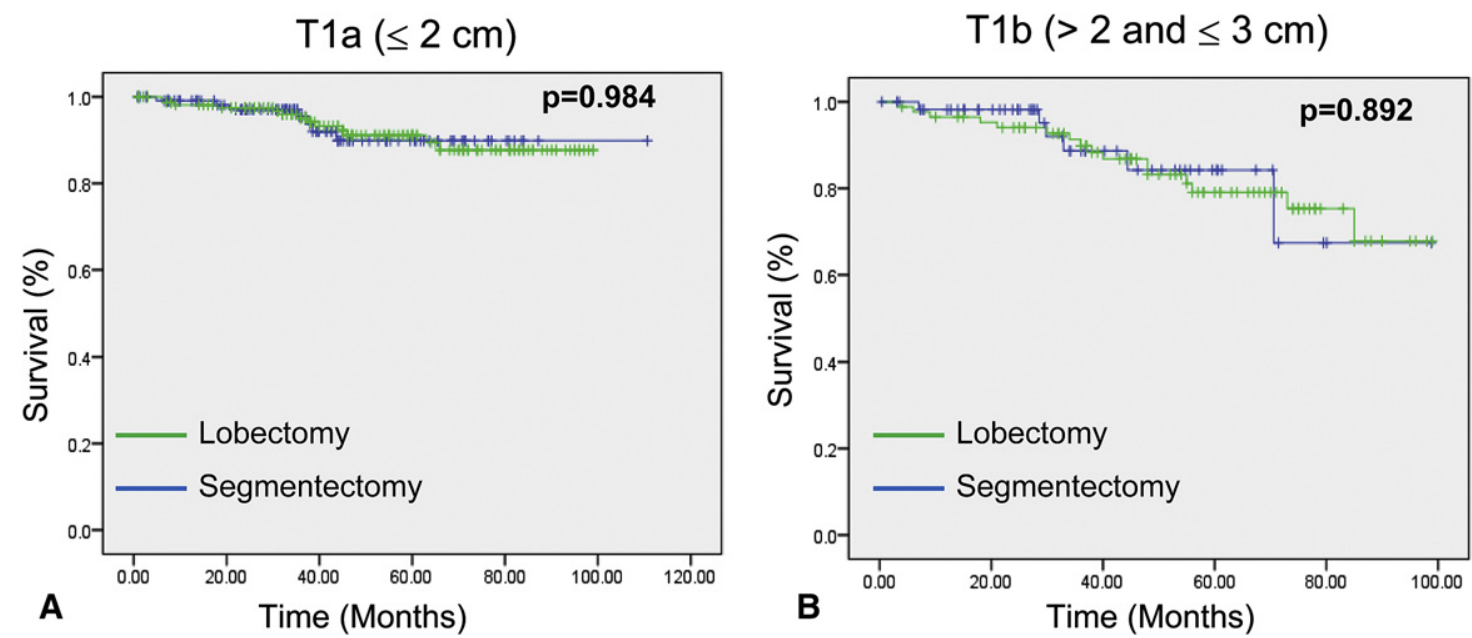

FIGURE 4. Cancer-specific survival curves comparing outcomes after segmentectomy or lobectomy for T1a (A) and T1b (B) tumors.

as Richard McElvein stated 20 years ago in a commentary on minimally invasive pulmonary surgery, most thoracic surgeons are now not familiar with this pulmonary parenchymal-sparing anatomic resection approach to benign or malignant pulmonary disease. ${ }^{18}$

The analysis of our experience with anatomic segmentectomy of both $\mathrm{T} 1 \mathrm{a}$ and $\mathrm{T} 1 \mathrm{~b}$ tumors within the overarching category of stage 1A NSCLC suggests that recurrencefree and cancer-specific survivals are equivalent among patients undergoing anatomic segmentectomy or lobectomy. Of course, careful mediastinal/hilar lymph node assessment to optimize the validation of this pathologic stage 1A disease status is mandatory to prevent the exclusion of patients with "surprise node positive" disease from the potential benefits of adjuvant chemotherapy and the necessarily erroneous assumptions of our surgical results with supposed stage $1 \mathrm{~A}$ disease. ${ }^{19-21}$

Another point of analytic importance relates to the noncancer-related mortalities among patients with stage 1A NSCLC in our experience. Non-cancer-related deaths seem to more common among patients undergoing segmentectomy as definitive surgical management for stage $1 \mathrm{~A}$ NSCLC, suggesting a continued selection bias in favor of lobectomy among thoracic surgeons for patients free of significant physiologic impairment.

So which direction are we to go with regard to the recommendations for the small peripheral stage 1A NSCLC? Should we be content in believing that lobectomy is the best approach for stage 1 NSCLC in these times of competitive pressures from nonsurgical image-guided approaches to "early-stage" NSCLC? ${ }^{22}$ Whatever the future brings regarding persistence or changes in therapeutic paradigms, we continue to recommend adherence to the primary surgical contentions of complete "R0" resection with negative surgical margins and complete nodal staging of the malignant disease.

\section{CONCLUSIONS}

Can we make the step forward away from the empirical recommendation of lobectomy and possibly toward selective consideration for anatomic segmentectomy when "anatomically feasible" for the patient with peripheral stage 1A NSCLC? The results of our experience suggest this is possible. However, the results of currently active prospective, randomized trials (Cancer and Leukemia Group B 140503) and possibly future "propensity matched trials" evaluating the utility of lobectomy compared with segmentectomy (and for that matter, image-guided ablative approaches to stage 1A NSCLC) will direct the appropriate clinical pathways for the ongoing management of earlystage NSCLC.

The authors thank Alicia Oostdyk, Peg Reamer, and Lorraine Ickes of the Thoracic Surgery Tumor Registry for assistance in database management and analysis; and Kimberly Burke, Nicole Larson, Lauren Matteo, Kimberly Swendsen, Patricia Williams, and Maria Haffely for assistance in organizing and analyzing hospital charts and providing timely updates regarding patient follow-up.

\section{References}

1. Goldstraw P, Crowley J, Chansky K, Giroux DJ, Groome PA, Rami-Porta R, et al. The IASLC Lung Cancer Staging Project: proposals for the revision of the TNM stage groupings in the forthcoming (seventh) edition of the TNM Classification of malignant tumours. $J$ Thorac Oncol. 2007;2:706-14.

2. Detterbeck FC, Boffa DJ, Tanoue LT, Wilson LD. Details and difficulties regarding the new lung cancer staging system. Chest. 2010;137:1172-80.

3. Edge SB, Byrd DR, Compton CC, Fritz AG, Greene FL, Trotti A, eds. AJCC Cancer Staging Handbook. 7th ed. New York: Springer; 2009.

4. Ginsberg RJ, Rubinstein LV. Randomized trial of lobectomy versus limited resection for T1 N0 non-small cell lung cancer. Lung Cancer Study Group. Ann Thorac Surg. 1995;60:615-23.

5. Okada M, Nishio W, Sakamoto T, Uchino K, Yuki T, Nakagawa A, et al. Effect of tumor size on prognosis in patients with non-small cell lung cancer: the role of segmentectomy as a type of lesser resection. J Thorac Cardiovasc Surg. 2005; 129:87-93.

6. Martin-Ucar AE, Nakas A, Pilling JE, West KJ, Waller DA. A case-matched study of anatomical segmentectomy versus lobectomy for stage I lung cancer in high-risk patients. Eur J Cardiothorac Surg. 2005;27:675-9. 
7. Schuchert MJ, Pettiford BL, Pennathur A, Abbas G, Awais O, Close J, et al. Anatomic segmentectomy for stage I non-small-cell lung cancer: comparison of video-assisted thoracic surgery versus open approach. J Thorac Cardiovasc Surg. 2009;138:1318-25. e1.

8. Wisnivesky JP, Henschke CI, Swanson S, Yankelevitz DF, Zulueta J, Marcus S, et al. Limited resection for the treatment of patients with stage IA lung cancer. Ann Surg. 2010;251:550-4.

9. El-Sherif A, Gooding WE, Santos R, Pettiford B, Ferson PF, Fernando HC, et al Outcomes of sublobar resection versus lobectomy for stage I non-small cell lung cancer: a 13-year analysis. Ann Thorac Surg. 2006;82:408-16.

10. Mery CM, Pappas AN, Bueno R, Colson YL, Linden P, Sugarbaker DJ, et al. Similar long-term survival of elderly patients with non-small cell lung cancer treated with lobectomy or wedge resection within the surveillance, epidemiology, and end results database. Chest. 2005;128:237-45.

11. Landreneau RJ, Sugarbaker DJ, Mack MJ, Hazelrigg SR, Luketich JD, Fetterman L, et al. Wedge resection versus lobectomy for stage I (T1 N0 M0) non-small-cell lung cancer. J Thorac Cardiovasc Surg. 1997;113:691-700.

12. Swanson SJ. Video-assisted thoracic surgery segmentectomy: the future of surgery for lung cancer? Ann Thorac Surg. 2010;89:S2096-7.

13. Santos R, Colonias A, Parda D, Trombetta M, Maley RH, Macherey R, et al. Comparison between sublobar resection and 125Iodine brachytherapy after sublobar resection in high-risk patients with stage I non-small-cell lung cancer. Surgery. 2003;134:691-7.

14. Churchill ED, Belsey R. Segmental pneumonectomy in bronchiectasis: the lingula segment of the left upper lobe. Ann Surg. 1939;109:481-99.

15. Jensik RJ, Faber LP, Milloy FJ, Monson DO. Segmental resection for lung cancer. A fifteen-year experience. J Thorac Cardiovasc Surg. 1973;66:563-72.

16. Overholt RH, Neptune WB, Ashraf MM. Primary cancer of the lung. A 42-year experience. Ann Thorac Surg. 1975;20:511-9.

17. Read RC, Yoder G, Schaeffer RC. Survival after conservative resection for T1 N0 M0 non-small cell lung cancer. Ann Thorac Surg. 1990;49:391-400.

18. McElvein RB. Invited commentary. Ann Thorac Surg. 1991;51:977-8.

19. Arriagada R, Bergman B, Dunant A, Le Chevalier T, Pignon JP, Vansteenkiste J. Cisplatin-based adjuvant chemotherapy in patients with completely resected non-small-cell lung cancer. N Engl J Med. 2004;350:351-60.

20. Winton T, Livingston R, Johnson D, Rigas J, Johnston M, Butts C, et al. Vinorelbine plus cisplatin vs observation in resected non-small-cell lung cancer. $N$ Engl J Med. 2005;352:2589-97.

21. Douillard JY, Rosell R, De Lena M, Carpagnano F, Ramlau R, GonzalesLarriba JL, et al. Adjuvant vinorelbine plus cisplatin versus observation in patients with completely resected stage IB-IIIA non-small-cell lung cancer (Adjuvant Navelbine International Trialist Association [ANITA]): a randomised controlled trial. Lancet Oncol. 2006;7:719-27.

22. Fernando HC, Schuchert M, Landreneau R, Daly BT. Approaching the high-risk patient: sublobar resection, stereotactic body radiation therapy, or radiofrequency ablation. Ann Thorac Surg. 2010;89:S2123-7.

\section{Discussion}

Dr Paul Schipper (Portland, Ore). I appreciate what you and your coauthors have done to evaluate the surgical treatment of these early-stage tumors. Your historical data applied to the new system get information out there quicker. However, you have to be cautious when you are making prospective or clinical decisions using retrospective pathologic data.

I have 2 questions for you based on the strong bias that exists when you do this. When we are in the operating room, we are usually working with a combination of clinical and, if we are doing our job right, pathologic data, but we do not have that final story. We do not have the final pathology. Where most of the mystery seems to lie, at least in my practice, is with the nodes that are in the lung itself, the N1 nodes, the intraparenchymal nodes. So my first question is, if I encounter a lesion, a $1.9-\mathrm{cm}$ or T1a lesion, and same question for a $2.5-\mathrm{cm}$ or $\mathrm{T} 1 \mathrm{~b}$ lesion, $\mathrm{N} 2$ node negative, good lung function, should I do a segment or a lobe? Before you answer, if on that final pathology I find there are N1 nodes that are positive and I did a segment, have I done the right operation?
Dr Carr. Let's start with the T1a lesion that is N2 negative. It is our standard that we actually dissect out the N1 nodes associated with the bronchovascular structures just before dividing them and send them off as frozen. When we get those back and there is a negative frozen section on that N1 lymph node, we then proceed immediately with an extended segmentectomy, as has been mainly described in the Japanese literature (Okada). However, if that N1 node comes back positive, we immediately assume there may be additional disease we are missing, which would obviously upstage that patient in terms of stage, and then proceed to lobectomy. The same is true then for the T1b tumors. We have the same approach to either patient. Identify N1 nodes, frozen section, and then if positive, the patient undergoes lobectomy as opposed to segmentectomy.

Dr Schipper. My second question then has to do with survivals. In the Abstract, you gave us recurrence-free survival and then today the additional information, which was very good, with the cancerspecific survivals. Things became a little more homogeneous when you went from one to the other. The trend that existed between better survival with lobectomy $(80 \%)$ and segmentectomy $(67 \%)$ in the T1b lesions, for example, disappeared when we looked at the cancer-specific survival. As you pointed out, that had to do with the fact that your patients undergoing segmentectomy were sicker. Your comparison slide showed that very well. They had worse $\mathrm{FEV}_{1}$, more chronic obstructive pulmonary disease, and more coronary artery disease. You then proved the patients undergoing segmentectomy were sicker by following them prospectively and finding they died sooner than those undergoing lobectomy and of cancer-unrelated causes. Because they died of these other things, the segmentectomy cases did not have the same opportunity as the lobectomy cases to die of lung cancer. It is a clinical decision that we make when I evaluate somebody, and I say I don't think you are going to live much longer for cancer-unrelated reasons, and therefore I am not going to give you what I perceive as the durable operation, lobectomy; rather, I am going to give you a less durable operation but one that is may be just as good for your specific situation, and that is the segmentectomy.

Do your data show that segmentectomy is just as good or potentially better for somebody who has a couple of decades left, somebody who is not going to die of cancer-unrelated causes in the near future?

Dr Carr. Initially when we started enrolling patients and segmentectomies were performed, we were looking at patients who had compromised lung function, and we were doing everything we could to do a parenchymal-sparing operation to give them the best quality of life and postoperative pulmonary function test results. However, we have begun performing segmentectomies on younger patients who have normal pulmonary function test results and are doing well. As you see, you are absolutely right, and it is a limitation of looking at a retrospective database when you have a difference in pulmonary function test results and the patients are older, but the recurrence-free survival with a mean overall followup overall of 45.3 months allows us to say that it is likely that a true anatomic segmentectomy, and not just a sublobar resection but an anatomic segmentectomy, is equivalent to a lobectomy in a patient who has decades left to live.

Dr Frederic Grannis (Duarte, Calif). This meeting has had a remarkable focus on health care policy and the science of health care 
delivery, and this problem you have shown here is a case where you can cure the overwhelming majority of lung cancers, but the cases you are describing make up only $7 \%$ of all lung cancers in the United States. Where did these cases come from? Did they come from the screening program for computed tomography in Pittsburgh or from serendipity? Finally, when are we going to stop waiting for patients to develop symptoms of lung cancer, at which point they are almost never curable, and move forward to have a health care system where we proactively screen for these cancers?

Dr Carr. At Pittsburgh we do have a lung cancer screening program (PLuSS) similar to the Early Lung Cancer Action Program (Claudia Henschke) that does assist in identifying patients with early-stage disease. In western Pennsylvania, there are only 2 large institutions performing more advanced lung surgery like this, Allegheny and Pittsburgh, and Pittsburgh has more hospitals, so our catchment is significantly higher. We are out in the community working with the pulmonologists getting them to realize that we do need to refer patients sooner and not wait. We'll enroll them in a screening process because they do have nodules, and we will keep track of them. We have pulmonologists who are dedicated and interested in just following these pulmonary nodules, and when there are any changes they immediately refer them to us.

Your second question is a bit trickier. As time goes by, we will be able to use screening tools, potentially genomic analysis, to risk stratify patients as we get more robust data on screening programs for lung cancer. However, I think we are all aware that with changes in health care that may be coming down the pike and the cost associated with this, we are going to need to find a costeffective way to screen. Until we find a cost-effective way to accurately screen patients with good sensitivity and specificity, we are still falling short. Hopefully those days are coming.

Dr Grannis. I would just remind you that as time passes, 160,000 people die each year, and we do have a way to fund it, and that is medical monitoring lawsuits against the tobacco industry.

Dr Thomas Rice (Cleveland, Ohio). I would like to propose another conclusion to your study or an alternate: The best patients with the worst cancer have a survival similar to the worst patients with the best cancer. The problem is that this is a retrospective study with significant bias in the selection of patients with similar survival, which may be the result of that selection bias. To make a fair comparison, you have to compare similar patients. This retrospective study would benefit immensely from propensity scoring and then to compare matched groups. That would be beneficial to your readers and a great addition to the literature.

Another comment is the lymph node harvest. People who undergo VATS have a mean and standard deviation that are equal. Those are skewed data, and it is better to present that as a median plus a range. How did you handle that statistically to compare those 2 groups? I think it has a bearing on recurrence.

Dr Carr. We agree that this is not level 1 evidence, and that these findings will require validation by a prospective, randomized study such as Dr Altorki's trial (CALGB 140503).

As far as the lymph nodes are concerned, we have found that the larger specimen associated with lobectomy produces higher lymph node counts of final pathologic examination. The total number of lymph node stations assessed was similar between the groups. Importantly, there is no level 1 evidence to suggest that doing a more aggressive lymph node dissection improves recurrence rates or survival following lung resection, as demonstrated by the findings of the ACOSOG Z0030 study.

Dr Joseph Shrager (Stanford, Calif). You end up concluding that up to $3 \mathrm{~cm}$ is okay for a sublobar resection. There are a couple of previous reports, I think you alluded to one of them, and I apologize that I do not remember where they each came from, with one suggesting that the margin has to be at least as large as the diameter of the tumor and one suggesting the margin has to be at least $2 \mathrm{~cm}$. You did not address anything about margins. Would you stand by those sorts of recommendations? In other words, when is a sublobar resection not appropriate?

Dr Carr. We have previously examined the issue of surgical margins in the context of segmentectomy and found that the risk of recurrence is diminished when surgical margin is greater than the size of the tumor (magin:tumor ratio $>1$ ). So a $2-\mathrm{cm}$ tumor would ideally be served by a resection with a $2-\mathrm{cm}$ margin. Interestingly, in the current analysis we did not see any significant difference in surgical margin:tumor diameter ratios when comparing lobectomy with segmentectomy for either T1a or T1b tumors, suggesting that we are intuitively selecting the surgery that permits adequate margins on an individual case-by-case basis. If we believe that the surgical margin would be compromised with an anatomic segmentectomy, than lobectomy should be preformed if the patient's physiologic status permits. 